\title{
Advantage of Delivering the Gene Therapy Tool (Cas9) as Purified Protein Formulation
}

Doolaanea AA*

Advanced Drug Delivery Lab, Department of Pharmaceutical Technology, Kulliyyah of Pharmacy, International Islamic University Malaysia, 25200 Kuantan, Malaysia

\begin{abstract}
Gene editing permits the alteration of genomic sequences in vitro and in vivo, which could serve as a powerful tool in basic research and human gene therapy. The recent clustered regularly interspaced short palindromic repeat (CRISPR) system has revolutionized the way of gene editing. To be used in human, a suitable in vivo-compatible delivery form and method should be used. The delivery form here is meant 'what' is being introduced to the body: plasmid DNA, viral vector, mRNA, purified protein or other while the delivery method is meant 'how' the CRISPR system is introduced or administered: lipid transfection, electroporation, nanoparticles, liposomes or others. The brief discussion in this article suggests that delivering the protein component of the CRISPR system as purified protein has several advantages considering different sides like the industrial feasibility and pharmacokinetic/ pharmacodynamic aspects.
\end{abstract}

Keywords: CRISPR; Cas9; Gene editing; Gene therapy; Formulation; Delivery

\section{Introduction}

Gene editing permits the alteration of genomic sequences in both cultured cells and organisms, which could serve as a powerful tool in basic research as well as have the potential in the application of gene therapy. Introducing a DNA double-stranded break (DSB) by a nuclease enzyme at the targeted site is the first step in precise gene editing. The early genome editing utilized DSB-inducing nucleases such as meganucleases, zinc finger nucleases, and transcription activator-like effector nucleases (TALENs). Recently, the more convenient clustered regularly interspaced short palindromic repeat (CRISPR) system has revolutionized the way of gene editing [1].

Gene editing tools such as CRISPR system have high potential to treat gene disorders and currently-untreatable diseases. However, to be used in human, a suitable in vivo-compatible delivery form and method should be used. The delivery form here is meant 'what' is being introduced to the body: plasmid DNA, viral vector, mRNA, purified protein or other. The delivery method is meant 'how' the CRISPR system is introduced or administered: lipid transfection, electroporation, nanoparticles, liposomes or others. This short article briefly discussed the advantages of delivering the protein component of the CRISPR system as purified protein considering different aspects like the industrial and pharmacokinetic/pharmacodynamics considerations.

\section{CRISPR System for Gene Editing}

The Clustered Regularly Interspaced Short Palindromic Repeats (CRISPR) and CRISPR Associated (Cas) system was first discovered in bacteria and functions as a defense against foreign DNA, either viral or plasmid $[2,3]$.

CRISPR system is currently the most commonly used RNA-guided endonuclease technology for genome engineering. There are two essential components in CRISPR system:

- A guide RNA

- An endonuclease, in this case the CRISPR associated (Cas) nuclease, Cas9.

When the gRNA and the Cas9 are present in the same cell, the genomic target sequence can be modified or permanently disrupted. For all gene editing applications, the two biomolecules i.e. Cas9 protein and gRNA (nucleic acid) must be introduced into each target cell. These two biomolecules form a complex with the DNA, specifically targeting DNA sites complementary to an approximately 20-base sequence within the gRNA and next to a proto-spacer adjacent motif (PAM). The identity of PAM sequence is defined by the particular Cas9 protein employed. The most commonly used Cas9 to date is from Streptococcus pyogenes, wherein the optimal PAM sequence is NGG (where ' $N$ ' is any nucleobase) [4].

\section{CRISPR Delivery Forms}

CRISPR system is currently delivered into the cell in different forms, by utilizing different delivery methods. The final aim of all forms of CRISPR system is to get both Cas9 (an endonuclease enzyme, a protein) and the gRNA (a nucleic acid) inside the cell.

CRISPR was delivered into the cell in form of:

- $\quad$ Plasmid DNA encoding Cas9 and gRNA [5],

- $\quad$ DNA template encoding Cas9 and gRNA [6],

- Viral vector encoding Cas9 and gRNA [2],

- $\quad$ mRNA to express Cas9, and gRNA [7];

- $\quad$ Recombinant Cas9 protein and gRNA [8],

- $\quad$ Some mixture of the previous forms like viral and non-viral [9].

As seen from previous forms, to get Cas9 inside the cell it is mostly given as DNA or mRNA that need to be first expressed in the cell. This involves a delivery of long sequence nucleic acid wherein

*Corresonding author: Doolaanea AA, Advanced Drug Delivery Lab, Department of Pharmaceutical Technology, Kulliyyah of Pharmacy, International Islamic University Malaysia, Malaysia, Tel: +60136238628; E-mail: abdalmonemdoolaanea@yahoo.com

Received May 02, 2017; Accepted May 15, 2017; Published May 25, 2017

Citation: Doolaanea AA (2017) Advantage of Delivering the Gene Therapy Too (Cas9) as Purified Protein Formulation. J Formul Sci Bioavailab 1: 111.

Copyright: ( 2017 Doolaanea AA. This is an open-access article distributed under the terms of the Creative Commons Attribution License, which permits unrestricted use, distribution, and reproduction in any medium, provided the original author and source are credited. 
therapeutic response is not well-established in the clinical use. Very few DNA-based or mRNA-based therapeutics are available for clinical use. In addition, the use of viral vectors has the drawbacks of inducing immunity reactions. In contrast, proteins have long been used and approved as therapeutic agents and currently available for clinical use such as insulin, alteplase and several monoclonal antibodies [10].

In addition, delivering Cas9 as nucleic acid (DNA, mRNA or virus) needs time to start its effect because the cell needs first to express it. On the other hand, by delivering Cas 9 as ready-made purified protein, the gene editing action can start immediately. Kim [8] reported that chromosomal DNA was cleaved efficiently almost instantly after delivery of purified Cas9 while it took three days to reach an equivalent level of gene editing when Cas9 was expressed via plasmid transfection. In this research, the authors used electroporation to achieve the gene editing, which is not suitable method for in vivo use. It can be deduced that for efficient and fast effect of gene editing, Cas9 is best delivered as purified protein.

\section{CRISPR Delivery Methods}

In term of delivery methods, the delivery vehicle should be in vivo compatible if to be used later for gene editing in human or other organisms. The in vivo delivery of the Cas9 system remains challenging. In cultured cells, electroporation, nucleofection, and lipofectaminemediated transfection have been used to deliver plasmid DNA encoding Cas9-gRNA complexes through cell membranes. These methods are welcomed in the in vitro experiment but for the application in vivo, physical delivery methods meet more challenges because of the variable physiological and pathological conditions [1]. Although viral carriers exhibited high efficiency in gene delivery and expression, viral delivery systems are generally concerned with their potential in carcinogenesis and immunogenicity [1]. In addition, viral vectors can only deliver the nucleic acid but not the purified Cas 9 .

Therapeutic proteins have been approved by United States Food and Drug Administration (FDA) for long time. Between 1982 and 2013, a total of 91 recombinant-protein-based new molecular entities (NMEs) gained FDA approval [10]. Thus, pharmaceutical industry has good experience with dealing with therapeutic proteins from research to production then clinical trials and getting approvals. On the other hand, dealing with long sequence nucleic acids (Cas9 plasmid or mRNA) might be challenging since the production and purification is different from proteins.

In term of the pharmacokinetic/pharmacodynamic (PKPD) of the therapeutic biologics, it is easier to deal with proteins. If Cas 9 enzyme (protein) is delivered into the body, it will start activity and degradation like any therapeutic protein. So, the PKPD is well-understood. However, for plasmid, the expression of Cas9 will take time. Then it will continue until plasmid degrades. During the time that plasmid is present in the cell, Cas9 is produced (expressed) and degraded simultaneously. After plasmid degrades, Cas9 production stops and Cas9 is only subjected to degradation. So the PKPD becomes complicated. As a result, adjusting the dose and dose frequency becomes more difficult with high probability of getting high variations among people.

Lastly, it should be highlighted that several carriers can be used to deliver proteins like polymer microparticles [11], alginate microparticles [12], chitosan [13] etc. Some protein delivery carriers were commercialized like Lupron Depot (lyophilized microspheres containing leuprolide acetate). However, since gRNA needs to be delivered into the same cell, it is advised to select a carrier that can deliver both proteins and nucleic acids simultaneously. Several carriers were successfully used to deliver proteins and RNA (may not be simultaneously) such as chitosan nanoparticles [14], poly lactide-coglycolide (PLGA) nanoparticles [15,16] alginate particles [12,17] and others. For example, Wang [18] used bio-reducible lipid nanoparticles to encapsulate and deliver Cas9-gRNA into cultured human cells and achieved more than $70 \%$ gene editing. This carrier enabled also the delivery of the protein into mouse brain in vivo.

\section{Conclusion}

Gene editing tools such as CRISPR system have high potential to treat currently-untreatable disorders but a suitable human-compatible delivery form and method should be used. Here we propose that delivering Cas9 component of the CRISPR system as purified protein has several advantages over other forms such as nucleic acid (mRNA or plasmid) or viral vectors. The most advantages lay behind its being as protein whereby proteins have long history as successful biologic drugs, well-known methods for manufacturing and easily understood pharmacokinetics/Pharmaco-dynamics. Other than that, protein delivery carriers are also known and already commercialized.

\section{Acknowledgments}

This publication is supported by IIUM RESEARCH INITIATIVE GRANT (RIG) THEME (RIGS15-092-0092).

\section{References}

1. Li L, He ZY, Wei XW, Gao GP, Wei YQ (2015) Challenges in CRISPR/CAS9 delivery: Potential roles of nonviral vectors. Human Gene Therapy 26: 452-462.

2. Mali P, Yang L, Esvelt KM, Aach J, Guell M, et al. (2013) RNA-guided human genome engineering via Cas9. Science 339: 823-826.

3. Barrangou R, Marraffini LA (2014) CRISPR-Cas systems: Prokaryotes upgrade to adaptive immunity. Mol Cell 54: 234-244.

4. Graham DB, Root DE (2015) Resources for the design of CRISPR gene editing experiments. Genome Biology16: 260.

5. Shen B (2014) Efficient Gene Disruption in Diverse Strains of Toxoplasma gondii Using CRISPR/CAS9. mBio 5(3): e01114-14.

6. Lee AF, Lloyd KCK (2014) Conditional targeting of Ispd using paired Cas9 nickase and a single DNA template in mice. FEBS Open Bio 4: 637-642.

7. Kang $X$ (2016) Introducing precise genetic modifications into human 3PN embryos by CRISPR/Cas-mediated genome editing. Journal of Assisted Reproduction and Genetics 33: 581-588.

8. Kim S (2014) Highly efficient RNA-guided genome editing in human cells via delivery of purified Cas9 ribonucleoproteins. Genome Research 24: 1012-1019.

9. Yin $\mathrm{H}$ (2016) Therapeutic genome editing by combined viral and non-vira delivery of CRISPR system components in vivo. Nat Biotech 34: 328-333.

10. Kinch MS (2015) An overview of FDA-approved biologics medicines. Drug Discovery Today 20: 393-398.

11. Ansary RH (2016) Preparation, characterization and in vitro release study of BSA-loaded double-walled glucose-poly(lactide-co-glycolide) microspheres. Archives of Pharmacal Research: 1-15.

12. Zhang Z (2016) Protein encapsulation in alginate hydrogel beads: Effect of pH on microgel stability, protein retention and protein release. Food Hydrocolloids 58: $308-315$

13. Amidi M (2010) Chitosan-based delivery systems for protein therapeutics and antigens. Advanced Drug Delivery Reviews 62: 59-82.

14. McCullough KC (2014) Self-replicating Replicon-RNA Delivery to Dendritic Cells by Chitosan-nanoparticles for Translation In Vitro and In Vivo. Molecular Therapy-Nucleic Acids 3: e173

15. Feczkó T (2011) Optimization of protein encapsulation in PLGA nanoparticles. Chemical Engineering and Processing: Process Intensification. 50: 757-765. 
Citation: Doolaanea AA (2017) Advantage of Delivering the Gene Therapy Tool (Cas9) as Purified Protein Formulation. J Formul Sci Bioavailab 1: 111. doi: 10.4172/2577-0543.1000122

Page 3 of 3

16. Wang L, Hao Y, Li H, Zhao Y, Meng D, et al. (2015) Co-delivery of doxorubicin and siRNA for glioma therapy by a brain targeting system: angiopep-2-modified poly(lactic-co-glycolic acid) nanoparticles. Journal of Drug Targeting: 1-15.

17. Li T,Wang GD, Tan YZ, Wang HJ (2014) Inhibition of lymphangiogenesis of endothelial progenitor cells with VEGFR-3 siRNA delivered with PEI-alginate nanoparticles. International Journal of Biological Sciences 10: 160.

18. Wang M (2016) Efficient delivery of genome-editing proteins using bio reducible lipid nanoparticles. Proceedings of the National Academy of Sciences 113 2868-2873. 